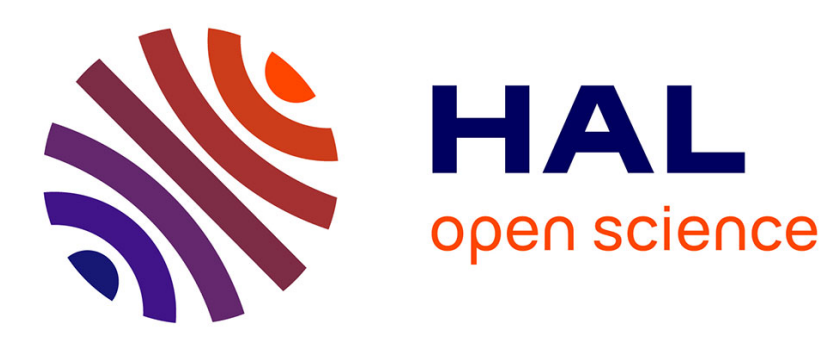

\title{
A new numerical strategy for the resolution of high-Péclet advection-diffusion problems
}

\author{
Pierre Ladevèze, Hervé Riou
}

\section{To cite this version:}

Pierre Ladevèze, Hervé Riou. A new numerical strategy for the resolution of high-Péclet advectiondiffusion problems. Computer Methods in Applied Mechanics and Engineering, 2012, 241-244, pp.302310. 10.1016/j.cma.2012.06.014 . hal-01647874

\section{HAL Id: hal-01647874 https://hal.science/hal-01647874}

Submitted on 17 Dec 2019

HAL is a multi-disciplinary open access archive for the deposit and dissemination of scientific research documents, whether they are published or not. The documents may come from teaching and research institutions in France or abroad, or from public or private research centers.
L'archive ouverte pluridisciplinaire HAL, est destinée au dépôt et à la diffusion de documents scientifiques de niveau recherche, publiés ou non, émanant des établissements d'enseignement et de recherche français ou étrangers, des laboratoires publics ou privés. 


\title{
A new numerical strategy for the resolution of high-Péclet advection-diffusion problems
}

\author{
H. Riou*, P. Ladevèze \\ LMT-Cachan (ENS Cachan/CNRS/Paris 6 University, PRES UniverSud Paris), 61 avenue du Président Wilson, F-94230 Cachan, France
}

This paper introduces a discontinuous method for the efficient determination of an approximate numerical solution of the two-dimensional advection-diffusion equation. Using the VTCR methodology (see [1]), this method involves free-space solutions of the governing partial differential equation. For the advection-diffusion equation with constant coefficients, the free-space solutions are exponential functions with a sharp gradient. The continuity of the solution across element boundaries is enforced weakly through a dedicated variational formulation. Preliminary results for a certain type of benchmark problem suggest that this approach is a promising numerical tool for handling such problems.

\section{Introduction}

The standard Galerkin finite element method (FEM) is the most popular numerical technique for the resolution of partial differential equations. However, since it approximates the solution using continuous, piecewise polynomial functions, it cannot handle problems involving sharp gradients unless the mesh is significantly refined. This is the case of advection-diffusion problems with high Péclet numbers, which are used to describe many transport phenomena and are involved in the linearization of the Navier-Stokes equations. Indeed, for such problems, it is well-known that the

\footnotetext{
* Corresponding author. Tel.: +33 147402238; fax: +33 147402240.

E-mail addresses: riou@lmt.ens-cachan.fr (H. Riou), ladeveze@lmt.ens-cachan.fr (P. Ladevèze).
}

solution presents rapid local variations in the boundary layer region (see Section 2.2 of [2]).

Numerical methods based on a modified FEM formulation have been proposed to solve this problem and stabilize the calculated solution. Some of these methods are the streamline upwind Petrov-Galerkin method [3,4], the Galerkin least-squares method $[5,6]$ and the unusual stabilized finite element method $[7,8]$. In these methods, the basis functions still remain polynomial.

Conversely, another approach consists in seeking a solution by adding new functions to the approximation space. Examples of such methods include the residual free bubbles method $[9,10]$, the partition of unity method [11] and the discontinuous enrichment method [12,13]. These methods have been applied efficiently to challenging examples. The method proposed in this paper belongs to this latter class of methods and distinguishes itself by 
the special shape functions it uses and by the treatment of the continuity equations at the interelement boundaries.

The formulation of the method is a direct extension of the variational theory of complex rays (VTCR) [1], which was developed for medium-frequency problems whose solutions have highly oscillating behavior. It is based on a dedicated variational formulation of the problem that allows the solutions inside the elements to be a priori independent of one another, thus giving the strategy great flexibility in the choice of shape functions. Then, one can use exact solutions of the governing equations as shape functions in each element. (In the case of medium-frequency acoustics and structural dynamics problems, these exact solutions are propagative and evanescent waves (see [1])). Such a choice drastically improves the efficiency of the method. The continuity of solutions at the interface between two elements is taken into account automatically through a dedicated variational formulation. In this paper, we present a numerical technique for advection-diffusion problems which preserves these characteristics.

The proven efficiency of the VTCR for structural vibration problems [14] and acoustic problems [15] was the main motivation behind this extension of the VTCR to advection-diffusion problems. The structure of the paper is the following: Section 2 presents the reference problem. Section 3 describes the variational formulation associated with the new approach. Section 4 is dedicated to the derivation of the shape functions. Section 5 illustrates the performance of the method through several numerical examples. Finally, the conclusions are presented in Section 6 .

\section{The reference problem}

Let $\Omega \subset R^{2}$ be a bounded open domain with boundary $\partial \Omega$, divided into $N_{E}$ non-overlapping elements $\Omega_{E}$ and their intersections $\Gamma_{E E^{\prime}}=\Omega_{E} \cap \Omega_{E^{\prime}}$. We consider the following advection-diffusion problem:

$$
\left\{\begin{array}{l}
\text { find } u_{E} \in H^{1}\left(\Omega_{E}\right) \text { such that : } \\
-\Delta u_{E}+\mathbf{a} \cdot \nabla u_{E}=0 \\
u_{E}=u_{d} \text { over } \partial \Omega_{E} \cap \partial \Omega \\
u_{E}=u_{E^{\prime}} \text { over } \quad \Gamma_{E E^{\prime}} \\
\nabla u_{E} \cdot \mathbf{n}_{E}+\nabla u_{E^{\prime}} \cdot \mathbf{n}_{E^{\prime}}=0 \text { over } \Gamma_{E E^{\prime}}
\end{array}\right.
$$

where $u_{d}$ is a prescribed boundary condition. a corresponds to the advection vector (e.g. the flow velocity vector in the context of fluid mechanics), which we assume to be constant and nonzero. In (1), the diffusivity parameter before $\Delta u$ was set to 1 , which does not affect the generality of this type of equation.

The Péclet number is a dimensionless parameter which relates the rate of advection of a flow to its rate of diffusion. Here, this parameter isequal to $l_{\Omega} \cdot\|\mathbf{a}\|$, where $l_{\Omega}$ is the characteristiclengthof $\Omega$.

\section{Variational formulation of the problem}

Let $S_{E, a d}$ denote the functional space of the functions $u_{E}$ which verify $-\Delta u_{E}+\mathbf{a} \cdot \nabla u_{E}=0$. Problem (1) is equivalent to the following 2-D variational formulation:

$$
\left\{\begin{array}{l}
\text { find } u_{E} \in S_{E, a d} \text { such that: } \\
\sum_{E} \int_{\partial \Omega_{E} \cap \partial \Omega}\left(u_{E}-u_{d}\right) \nabla v_{E} \cdot \mathbf{n}_{E} d \partial \Omega_{E}-\sum_{E} \int_{\partial \Omega_{E} \cap \partial \Omega} \frac{\mathbf{a} \cdot \mathbf{n}_{E}}{2}\left(u_{E}-u_{d}\right) v_{E} d \partial \Omega_{E} \\
+\frac{1}{2} \sum_{E} \sum_{E^{\prime}>E} \int_{\Gamma_{E E^{\prime}}}\left(u_{E}-u_{E^{\prime}}\right) \cdot\left(\nabla v_{E} \cdot \mathbf{n}_{E}-\nabla v_{E^{\prime}} \cdot \mathbf{n}_{E^{\prime}}\right) d \Gamma_{E E^{\prime}} \\
+\frac{1}{2} \sum_{E} \sum_{E^{\prime}>E} \int_{\Gamma_{E E^{\prime}}}\left(\nabla u_{E} \cdot \mathbf{n}_{E}+\nabla u_{E^{\prime}} \cdot \mathbf{n}_{E^{\prime}}\right) \cdot\left(v_{E}+v_{E^{\prime}}\right) d \Gamma_{E E^{\prime}} \\
-\sum_{E} \sum_{E^{\prime}>E} \int_{\Gamma_{E E^{\prime}}}\left(\frac{\mathbf{a} \cdot \mathbf{n}_{E}}{2} u_{E}+\frac{\mathbf{a} \cdot \mathbf{n}_{E^{\prime}}}{2} u_{E^{\prime}}\right) \cdot\left(v_{E}+v_{E^{\prime}}\right) d \Gamma_{E E^{\prime}}=0 \quad \forall v_{E} \in S_{E, a d}
\end{array}\right.
$$

or, in shorter form:

$$
\left\{\begin{array}{l}
\text { find } u \in S_{a d} \text { such that : } \\
a(u, v)=l(v) \quad \forall v \in S_{a d}
\end{array}\right.
$$

where $a(\cdot, \cdot)$ and $l(\cdot)$ are the bilinear form and the linear form of $(2)$ respectively.

In order to prove the equivalence of (1) and (2), one needs only to prove the uniqueness of (2), which the exact solution of (1) verifies trivially. Then, let us consider two different solutions of (2) and let $\delta u$ be their difference. By choosing $v=\delta u$ in (3), one gets:

$$
\sum_{E} \int_{\partial \Omega_{E}} \delta u_{E} \cdot \nabla \delta u_{E} \cdot \mathbf{n}_{E} d \partial \Omega_{E}-\sum_{E} \int_{\partial \Omega_{E}} \frac{\mathbf{a} \cdot \mathbf{n}_{E}}{2} \delta u_{E} \cdot \delta u_{E} d \partial \Omega_{E}=0
$$

Applying the Stokes formula, (4) leads to

$\sum_{E} \int_{\Omega_{E}} \nabla \delta u_{E} \cdot \nabla \delta u_{E}+\delta u_{E} \Delta u_{E} d \Omega_{E}-\sum_{E} \int_{\Omega_{E}} \mathbf{a} \cdot \nabla \delta u_{E} \cdot \delta u_{E} d \Omega_{E}=0$

Reverting to the definition of $S_{E, a d}$, one gets:

$\sum_{E} \int_{\Omega_{E}} \nabla \delta u_{E} \cdot \nabla \delta u_{E} d \Omega_{E}=0$

Then, $\boldsymbol{\nabla} \delta u_{E}=\mathbf{0}$ for every $\Omega_{E}$ and consequently $\delta u_{E}$ is constant.

Now let us consider an element $\boldsymbol{\Omega}_{E}$ such that $\partial \boldsymbol{\Omega}_{E} \cap \partial \boldsymbol{\Omega}=\emptyset$ (or choose for $\Omega_{E}$ an interior element of $\Omega$ ), and take test functions $v$ to be equal to zero everywhere in $\Omega$, except in $\Omega_{E}$. Since, according to (6), $\delta u_{E}$ and $\delta u_{E^{\prime}}$ are constant, (2) becomes

$\int_{\partial \Omega_{E}}\left(\nabla v_{E} \cdot \mathbf{n}_{E}-v_{E} \mathbf{a} \cdot \mathbf{n}_{E}\right) \cdot\left(\delta u_{E}-\delta u_{E^{\prime}}\right) d \partial \Omega_{E}=0 \forall v_{E} \in S_{E, a d}$

This problem can be written as $\int_{\partial \Omega_{E}} z .\left(\delta u_{E}-\delta u_{E^{\prime}}\right) d \partial \Omega_{E}=0 \forall z$ such that $z=\nabla v_{E} \cdot \mathbf{n}_{E}-v_{E} \mathbf{a} \cdot \mathbf{n}_{E}$ with $v_{E} \in S_{E, a d} . z$ must satisfy some conditions.

More precisely, the problem defined by $v_{E} \in S_{E, a d}$ and $z=\nabla v_{E}$ $\cdot \mathbf{n}_{E}-v_{E} \mathbf{a} \cdot \mathbf{n}_{E}$ over $\partial \Omega_{E}$ can be written in the following weak form: $\left\langle v_{E}, w\right\rangle=\int_{\Omega_{E}}\left(\nabla v_{E}-\mathbf{a} v_{E}\right) \cdot \nabla w d \Omega_{E}=\int_{\partial \Omega_{E}} z w d \partial \Omega_{E} \forall w \in H^{1}\left(\Omega_{E}\right)$. The mathematical kernel of this problem may be spanned by a set of functions $\varphi_{i}, i=1 \ldots m$ which verify $\left\langle v_{E}, \varphi_{i}\right\rangle=0 \forall v_{E} \in H^{1}\left(\Omega_{E}\right){ }^{1}$ Consequently, a necessary condition which $z$ must satisfy is $\int_{\partial \Omega_{\mathrm{E}}} z \varphi_{i} d \partial \Omega_{E}=0, i=1 \ldots m$.

Finally, going back to Problem (7), one can write $\int_{\partial \Omega_{E}} z$. $\left(\delta u_{E}-\delta u_{E^{\prime}}\right) d \partial \Omega_{E}=0 \quad \forall z$ such that $\int_{\partial \Omega_{E}} z \varphi_{i} d \partial \Omega_{E}=0, i=1 \ldots m$. Introducing the constant Lagrange multipliers $\lambda_{i}$, this equality under constraints becomes $\int_{\partial \Omega_{E}} z \cdot\left(\delta u_{E}-\delta u_{E^{\prime}}\right) d \partial \Omega_{E}+\lambda_{i} \int_{\partial \Omega_{E}} z \varphi_{i} d$ $\partial \boldsymbol{\Omega}_{E}=0 \forall z \in L^{2}\left(\partial \boldsymbol{\Omega}_{E}\right)$. It follows that $\delta u_{E}-\delta u_{E^{\prime}}+\sum_{i=1 \ldots m_{i}} \lambda_{i} \varphi_{i}=0$. Since $\varphi_{i}$ are continuous functions, the elements $\Omega_{E^{\prime}}$ surrounding $\Omega_{E}$ have the same $\delta u_{E^{\prime}}$.

Using a similar proof, one can show that if $\partial \Omega_{E} \cap \partial \Omega \neq \emptyset$ then $\delta u_{E}+\sum_{i=1 \ldots m} \lambda_{i} \varphi_{i}=0$ over $\partial \Omega_{E} \cap \partial \Omega$ and $\sum_{i=1 \ldots m} \lambda_{i} \varphi_{i}=0$ over the complementary part of $\partial \Omega_{E}$. Therefore, $\delta u_{E}=0$.

This proves that (2) is unique and equivalent to (1).

Several remarks can be made:

- Formulation (2) is a direct extension of the VTCR formulation introduced in [15] for Helmholtz problems, but adapted to advection-diffusion problems.

- (2) is not obtained by multiplying the governing equation of (1) by a test function and integrating the result by parts. Therefore, this formulation is not a classical Galerkin method.

\footnotetext{
${ }^{1}$ One can see that $\varphi_{i}$ verifies $\int_{\Omega_{E}}\left(\nabla v_{E}-\mathbf{a} v_{E}\right) \cdot \nabla \varphi_{i} d \Omega_{E}=0 \forall v_{E} \in H^{1}\left(\Omega_{E}\right)$. Then, according to the Stokes formula, $\varphi_{i}$ also verifies $\int_{\partial \Omega_{E}} v_{E} \cdot \nabla \varphi_{i} \cdot \mathbf{n}_{E} d \partial \Omega_{E}-\int_{\Omega_{E}} v_{E} \cdot \Delta \varphi_{i} d \Omega_{E}-\int_{\Omega_{E}}\left(\mathbf{a} v_{E}\right) \cdot \nabla \varphi_{i} d \Omega_{E}=0 \forall v_{E} \in H^{1}\left(\Omega_{E}\right)$. Consequently, one has $\Delta \varphi_{i}+\mathbf{a} \cdot \nabla \varphi_{i}=0$ within $\Omega_{E}$ and $\nabla \varphi_{i} \cdot \mathbf{n}_{\mathbf{E}}=0$ over $\partial \Omega_{E}$. This shows that $\varphi_{i}$ are continuous functions.
} 
- No Lagrange multiplier is required to ensure the continuity of the solution across the interface $\Gamma_{E E^{\prime}}$. This property is granted automatically by the variational formulation (2). This marks the difference between our approach and that of [16].

- The equivalence of (1) and (2) remains true even if the advection vector a is not constant as long as it is divergence-free, which is often the case in engineering applications (see Section 2.1 of [2]). Future developments of the technique presented here will address this case.

\section{Determination of the space of the shape functions}

In order to find an approximate solution of (1) the basic idea consists in seeking a solution of (3) in a space $S_{E, a d}^{q} \subset S_{E, a d}$ of finite dimension:

$$
\left\{\begin{array}{l}
\text { find } u^{q} \in S_{a d}^{q} \text { such that : } \\
a\left(u^{q}, v^{q}\right)=l\left(v^{q}\right) \quad \forall v^{q} \in S_{a d}^{q}
\end{array}\right.
$$

Then, in order to find some functions of $S_{E, a d}^{q}$, one must solve the equation $-\Delta u_{E}+\mathbf{a} \cdot \nabla u_{E}=0$. Since throughout this paper $\mathbf{a}$ is assumed to be constant and nonzero, the solution of (1) is known to have sharp exponential gradients. Therefore, we seek solutions in the form of $e^{\mathbf{k} \cdot \mathbf{x}}$ functions. The easiest way to obtain the vector $\mathbf{k}$ is to write it as $\mathbf{k}=\frac{\mathbf{a}}{2}+\mathbf{k}^{\prime}$, as was proposed in [16]. Indeed, when this expression is injected into the equation $-\Delta u_{E}+\mathbf{a} \cdot \nabla u_{E}=0$, one gets $-\left\|\mathbf{k}^{2}\right\|+\mathbf{a} \cdot \mathbf{k}=0$, which leads to
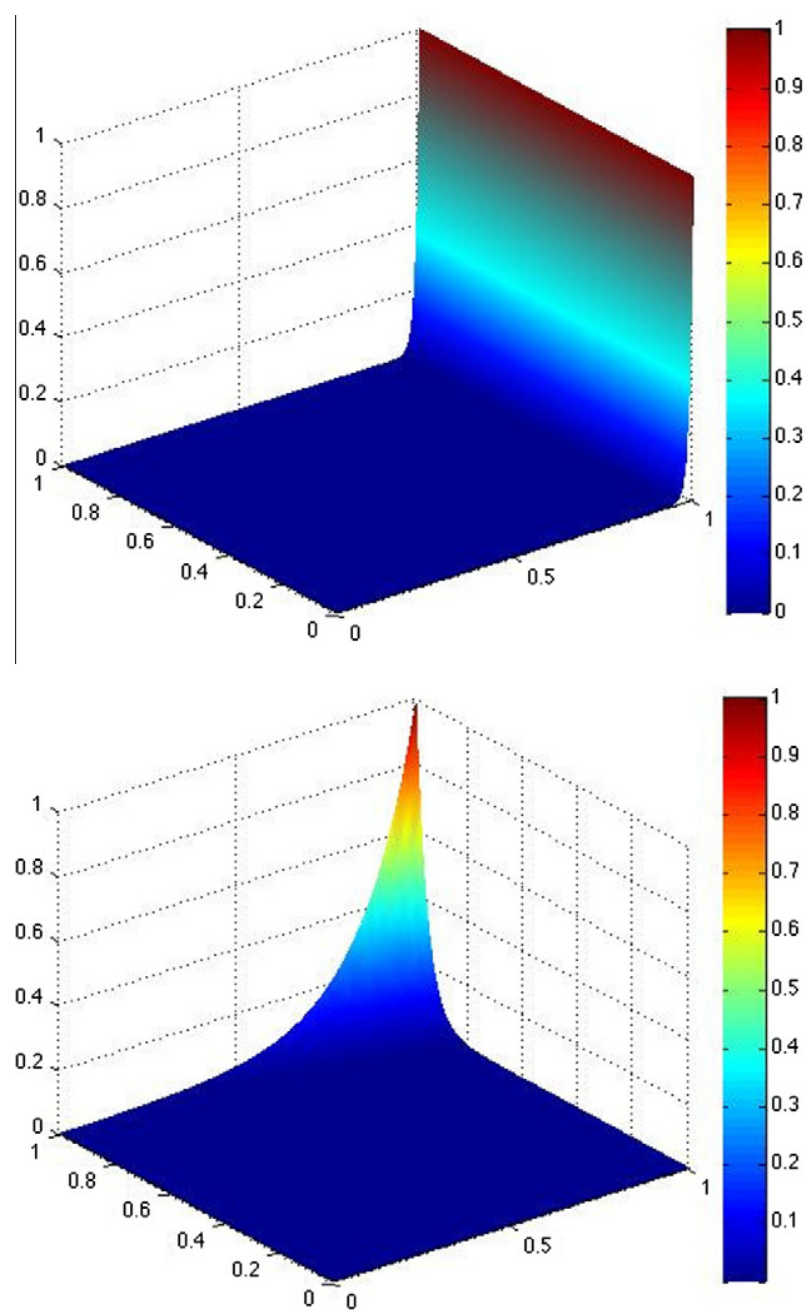

$\left\|\mathbf{k}^{\prime}\right\|^{2}=\frac{\|\mathbf{a}\|^{2}}{4}$

Then, the test functions $e^{\mathbf{k} \cdot \mathbf{x}}$ in $S_{E, a d}^{q}$ are taken such that

$\mathbf{k}(\theta)=\frac{\mathbf{a}}{2}+\frac{\|\mathbf{a}\|}{2}(\cos (\psi+\theta) \mathbf{x}+\sin (\psi+\theta) \mathbf{y})$

where $\psi$ and $\theta$ are respectively the direction of $\mathbf{a}$ and the polar direction in the $(\mathbf{x}, \mathbf{y})$ 2-D plane. These test functions are shown in Fig. 1. One can observe that the governing equation has a solution which is a constant function and that (10) allows the existence of such a function by choosing $\theta=\pi$.

However, the functions $e^{\mathbf{k} \cdot \mathbf{x}}$ grow exponentially in some direction. In order to avoid numerical problems with the conditioning number of the matrix system due to the possible very high values taken by these functions, it is preferable to normalize each shape function. Thus, one can use a specific origin $\mathbf{x}_{0}$ in the exponential function: $e^{\mathbf{k} \cdot\left(\mathbf{x}-\mathbf{x}_{0}\right)}$. Such an origin can be located at the boundary of $\Omega_{E}$ and chosen such that $\mathbf{k} \cdot\left(\mathbf{x}-\mathbf{x}_{0}\right) \leqslant 0 \forall \mathbf{x} \in \Omega_{E}$. Consequently, all the shape functions have almost identical maximum values. This property was used in Fig. 1.

As was explained well in [17], there are several ways to span $S_{E, a d}^{q}$. The first option is to use

$S_{E, a d}^{q}=\operatorname{span}\left\{e^{\mathbf{k}\left(\theta_{n}\right) \cdot\left(\mathbf{x}-\mathbf{x}_{\mathbf{0}}\right)}, \theta_{n}=\frac{2 \pi n}{q}, n=0 \ldots q-1\right\}$
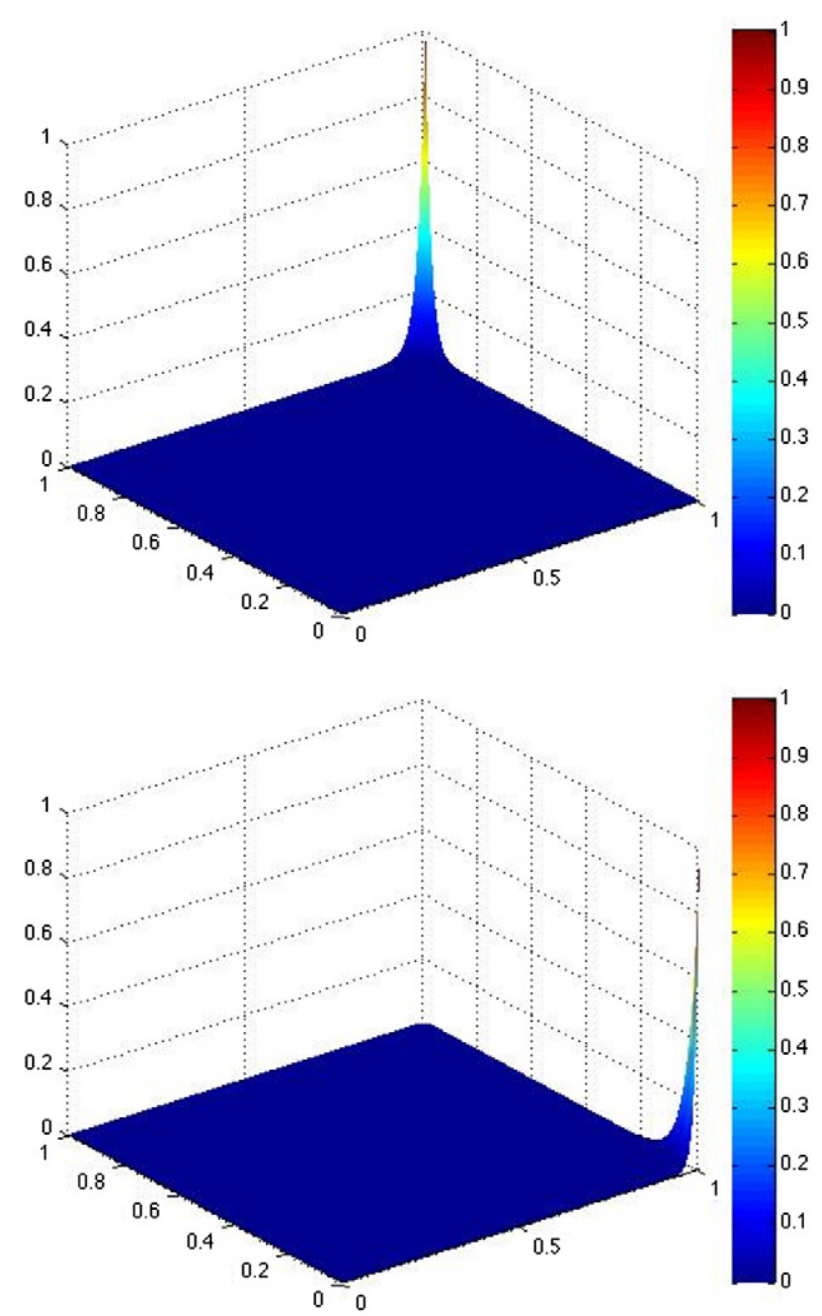

Fig. 1. The shape functions $e^{\mathbf{k}(\theta) \cdot\left(\mathbf{x}-\mathbf{x}_{0}\right)}$, with $\mathbf{k}(\theta)$ defined by (10) for different values of $\theta: \theta=0$ (upper left), $\theta=\pi / 3$ (upper right), $\theta=5 \pi / 3$ (lower left) and $\theta=-\pi / 6$ (lower right), for $\psi=0$. 
This is an interesting choice when the elements have simple geometries: exponentials are easy to integrate and, therefore, the integrals in (2) can be calculated analytically without any numerical quadrature error. A second option can be $S_{E, a d}^{q}=\operatorname{span}\left\{\int_{\frac{2 \pi n}{q}}^{\frac{2 \pi(n+1)}{q}}\right.$ $\left.e^{\mathbf{k}(\theta) \cdot\left(\mathbf{x}-\mathbf{x}_{0}\right)} d \theta, n=0 \ldots q-1\right\}$. This choice can be interesting if the solution being sought has a continuous distribution of exponential functions in the $\theta$ direction in the 2-D plane. However, some integrations in (2) must still be performed numerically. A third possible option, proposed by Kovalevsky et al. [17], is $S_{E, a d}^{q}=\operatorname{span}\left\{\int_{0}^{2 \pi}\right.$ $\left.e^{i n \theta} e^{\mathbf{k}(\theta) \cdot\left(\mathbf{x}-\mathbf{x}_{0}\right)} d \theta, n=-q \ldots q\right\}$. This choice corresponds to a Fourier series expansion of the amplitudes of the exponential functions and has been shown to be very interesting in the context of medium-frequency problems (see [17]) but again can lead to the evaluation of hard integrals. In the scope of this paper which is a first development of this approach for advection-diffusion problems, we retained the first option (11) as we did not want any roundoff errors due to numerical integrations. With such a choice, the analytical integrations offered with (11) gives the insurance that the errors are only due to the choice of shape functions, and not to numerical implementation. The performances of the other options will be assessed in future works.

The uniqueness of $(8)$ is established provided that the properties required for the uniqueness of (2) hold. Because of the properties of (2) proven in Section 3, this is the case if the number of equations is sufficient. This influences the choice of $S_{E, a d}^{q}$ and depends on the type of problem being studied.

\section{Numerical results}

In this section, the performance of the numerical strategy presented in Sections 3 and 4 is assessed for three advection-diffusion problems. All three problems are defined on a unit square $\Omega=[0 ; 1] \times[0 ; 1]$ discretized uniformly using a $\frac{1}{h} \times \frac{1}{h}$ mesh with a total of $N_{E}=\frac{1}{h^{2}}$ elements.

\subsection{The boundary layer problem with a flow aligned with the advection direction}

Let us consider an advection-diffusion problem in which the Dirichlet boundary conditions along $\partial \boldsymbol{\Omega}$ are such that the exact solution is $u_{e x}=\frac{e^{\mathbf{a}\left(\mathbf{x}-\mathbf{x}_{0}\right)}-1}{e^{-\mathbf{a} \mathbf{x}_{0}}-1}$, with $\mathbf{x}_{0}=(1,1)$ and $\psi=\left(\mathbf{x}_{0}, \mathbf{a}\right) \in\{0, \pi / 6$,

Table 1

The relative error in $L^{2}$-norm for the problem defined in Section 5.1 and for different advection directions $\psi$ and different numbers of shape functions $q$ per element (see (11)).

\begin{tabular}{llllll}
\hline$\|\mathbf{a}\|$ & $\psi$ & $Q_{1}$ & $q=4$ & $q=8$ & $q=16$ \\
\hline \multirow{2}{*}{$10^{2}$} & 0 & $8.97 \times 10^{-2}$ & $1.60 \times 10^{-15}$ & $1.58 \times 10^{-15}$ & $6.68 \times 10^{-14}$ \\
& $\pi / 6$ & $1.31 \times 10^{-2}$ & $3.36 \times 10^{-15}$ & $3.52 \times 10^{-15}$ & $4.78 \times 10^{-14}$ \\
& $\pi / 5$ & $1.31 \times 10^{-2}$ & $2.33 \times 10^{-15}$ & $3.45 \times 10^{-15}$ & $3.65 \times 10^{-14}$ \\
& $\pi / 4$ & $1.31 \times 10^{-2}$ & $8 ; 95 \times 10^{-17}$ & $2.09 \times 10^{-15}$ & $5.35 \times 10^{-14}$ \\
& $\pi / 3$ & $1.31 \times 10^{-2}$ & $6.27 \times 10^{-15}$ & $6.18 \times 10^{-15}$ & $3.51 \times 10^{-14}$ \\
$10^{3}$ & 0 & $57.7 \times 10^{-2}$ & $3.23 \times 10^{-15}$ & $4.85 \times 10^{-15}$ & $6.57 \times 10^{-14}$ \\
& $\pi / 6$ & $2.53 \times 10^{-2}$ & $8.03 \times 10^{-15}$ & $1.35 \times 10^{-14}$ & $2.11 \times 10^{-14}$ \\
& $\pi / 5$ & $2.57 \times 10^{-2}$ & $3.94 \times 10^{-14}$ & $3.26 \times 10^{-11}$ & $3.27 \times 10^{-14}$ \\
& $\pi / 4$ & $2.62 \times 10^{-2}$ & $2.58 \times 10^{-14}$ & $2.64 \times 10^{-14}$ & $4.70 \times 10^{-14}$ \\
& $\pi / 3$ & $2.53 \times 10^{-2}$ & $5.38 \times 10^{-14}$ & $5.46 \times 10^{-14}$ & $5.57 \times 10^{-14}$ \\
$10^{6}$ & 0 & $8.44 \times 10^{2}$ & $3.56 \times 10^{-12}$ & $5.08 \times 10^{-12}$ & $1.54 \times 10^{-11}$ \\
& $\pi / 6$ & 9.75 & $8.04 \times 10^{-12}$ & $8.26 \times 10^{-9}$ & $6.25 \times 10^{-9}$ \\
& $\pi / 5$ & 9.97 & $6.60 \times 10^{-14}$ & $1.73 \times 10^{-12}$ & $1.74 \times 10^{-12}$ \\
& $\pi / 4$ & 9.97 & $2.60 \times 10^{-11}$ & $2.75 \times 10^{-11}$ & $3.00 \times 10^{-11}$ \\
& $\pi / 3$ & 9.75 & $1.42 \times 10^{-10}$ & $9.75 \times 10^{-7}$ & $4.07 \times 10^{-7}$ \\
\hline
\end{tabular}

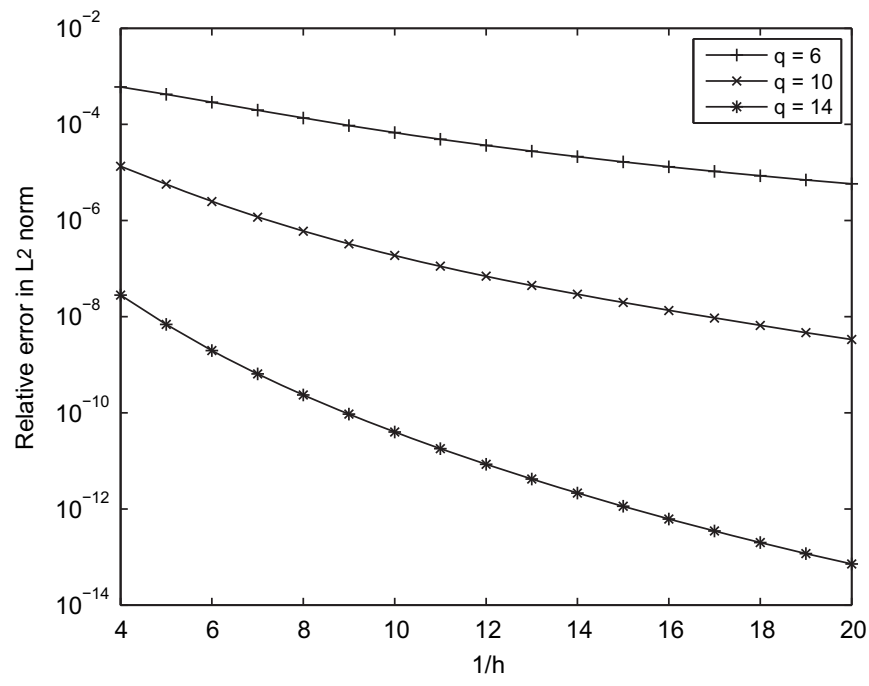

Fig. 2. $h$-convergence curves of the relative error in $L^{2}$-norm for the example of Section 5.2 for $\|\mathbf{a}\|=10^{2}$.

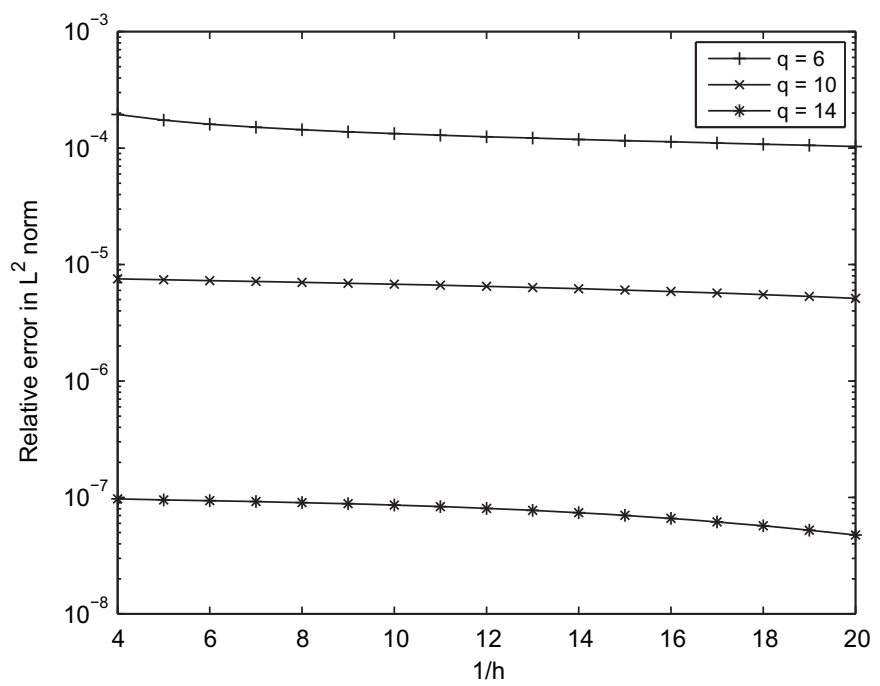

Fig. 3. $h$-convergence curves of the relative error in $L^{2}$-norm for the example of Section 5.2 for $\|\mathbf{a}\|=10^{3}$.

$\pi / 5, \pi / 4, \pi / 3\}$. This example was previously used in [18] for stabilized finite element methods and in [16] for the discontinuous enrichment method. Three different values of $\|\mathbf{a}\|\left(10^{2}, 10^{3}\right.$ and $10^{6}$ ) were tested.

Since (10) and (11) take into account the exponential term and the constant shape function, our approach was expected to lead to the exact solution within machine precision.

Table 1 shows the results, expressed as relative errors in terms of $L^{2}$-norm between $u^{q}$ and $u_{e x}$, compared to those of the classical Q1 finite element. ${ }^{2}$ As expected, the numerical solution is identical to the exact solution within machine precision. As already observed in [16], the shape functions described in (11) give a closer represen-

\footnotetext{
2 Alternative techniques cited in the introduction would give better results that the Q1 FEM. Here the FEM results must only be considered as order of magnitudes of the errors obtained with a classic method, without paying attention to numerical improvements for that class of problems. Furthermore, using finite elements with higher-degree polynomials would obviously lead to better results than the Q1 element, as shown in [20].
} 


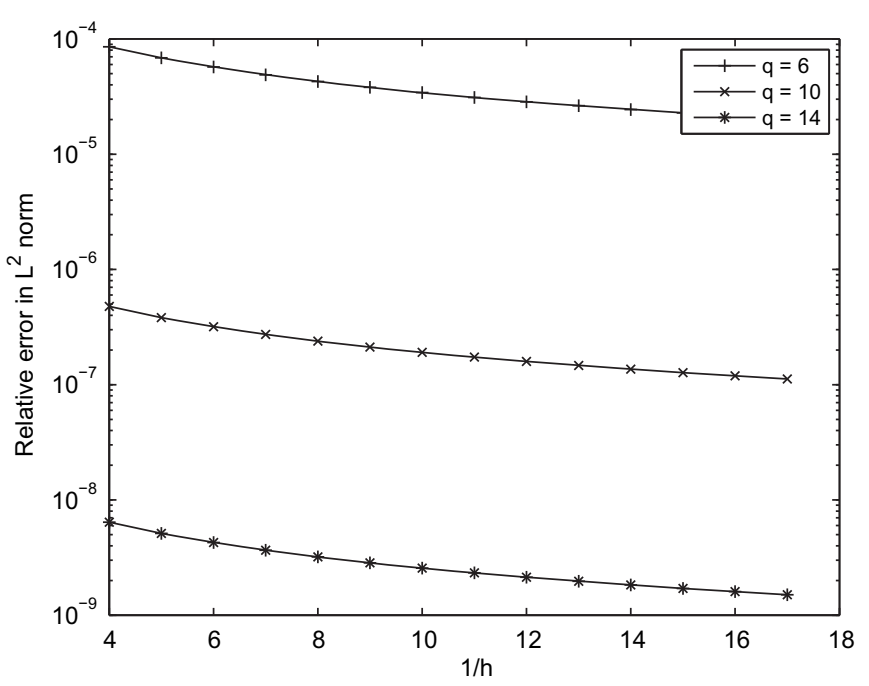

Fig. 4. $h$-convergence curves of the relative error in $L^{2}$-norm for the example of Section 5.2 for $\|\mathbf{a}\|=10^{6}$.

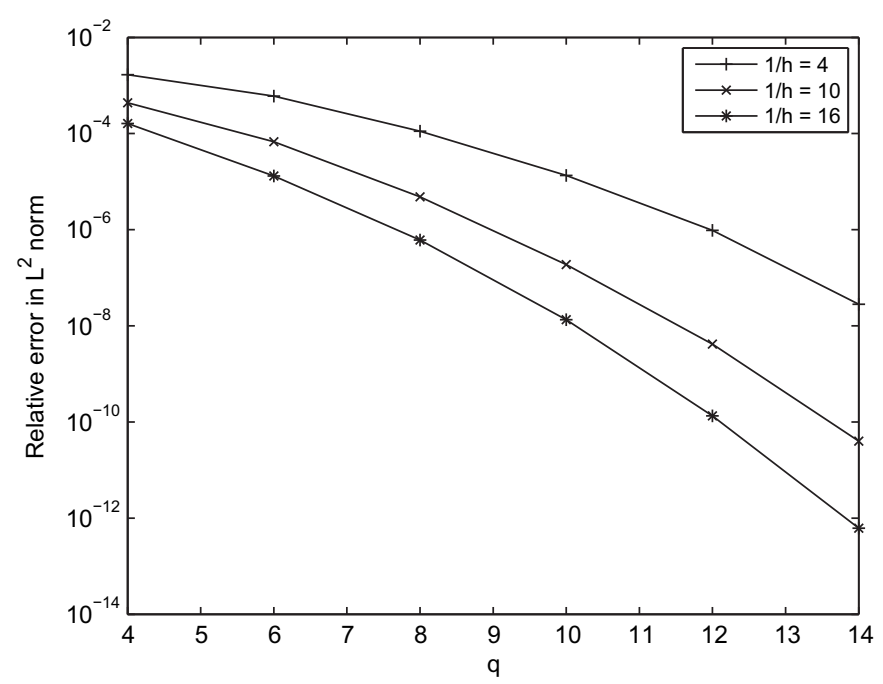

Fig. 5. $q$-convergence curves of the relative error in $L^{2}$-norm for the example of Section 5.2 for $\|\mathbf{a}\|=10^{2}$.

tation of the exact solution than the usual polynomial functional basis. Let us recall that the main difference between the approach proposed here and that of [16] is that no Lagrange multiplier is needed to enforce the continuity of the solution between elements. This continuity is automatically achieved in the variational formulation (2). Therefore, in our case, with a mesh of $N_{E}$ elements and $q$ shape functions per element, the total number of degrees of freedom is simply $N_{E} \times q$.

One can observe that for $\|\mathbf{a}\|=10^{6}$ our approach still outperforms the Galerkin $Q 1$ element, but sometimes fails to achieve machine precision. This is due to numerical difficulties: for such a value of $\|\mathbf{a}\|$, the matrix associated with (3) can be poorly conditioned. Nevertheless, the accuracy of the final result remains quite acceptable.

\subsection{The boundary layer problem with a flow not aligned with the advection direction}

Now let us consider the advection-diffusion problem where the Dirichlet boundary conditions over $\partial \Omega$ are such that the exact

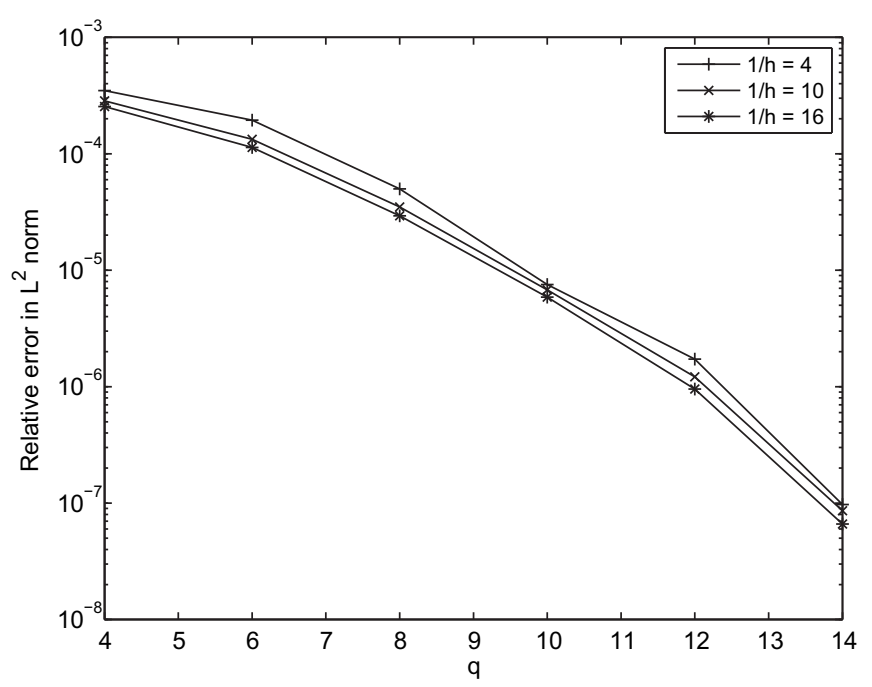

Fig. 6. $q$-convergence curves of the relative error in $L^{2}$-norm for the example of Section 5.2 for $\|\mathbf{a}\|=10^{3}$.

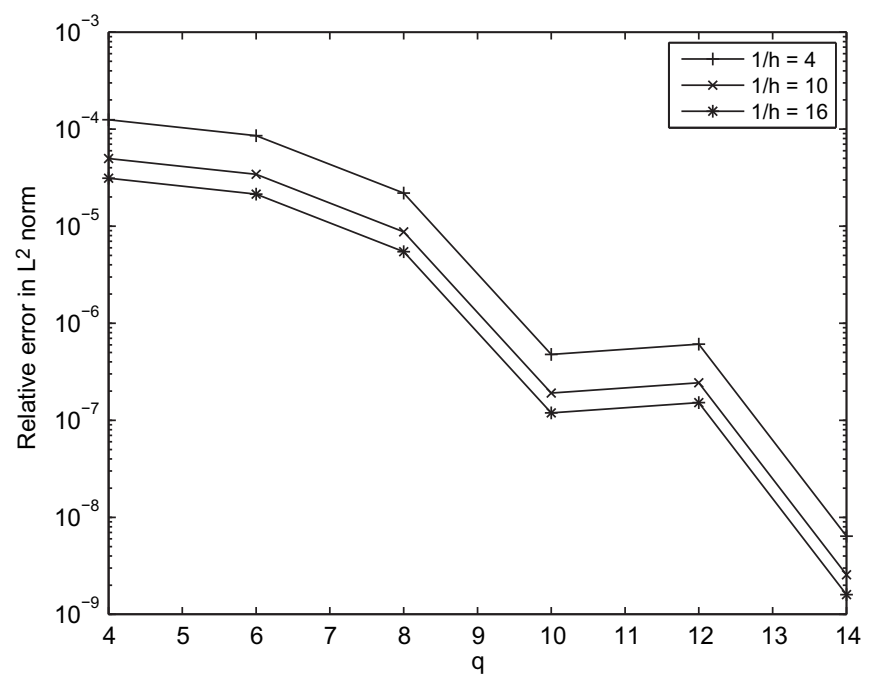

Fig. 7. $q$-convergence curves of the relative error in $L^{2}$-norm for the example of Section 5.2 for $\|\mathbf{a}\|=10^{6}$.

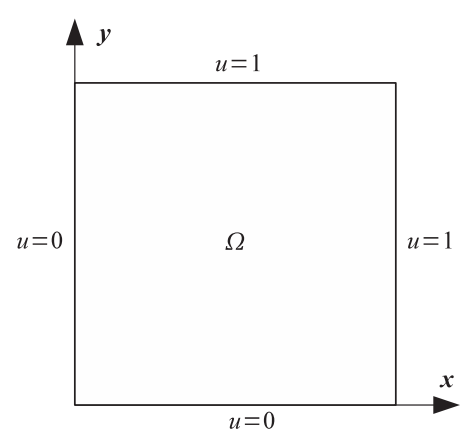

Fig. 8. Definition of the problem considered in Section 5.3. The advection vector is $\mathbf{a}=(a, 0)$. Two cases are considered: $\|\mathbf{a}\|=10^{2}$ and $\|\mathbf{a}\|=10^{3}$.

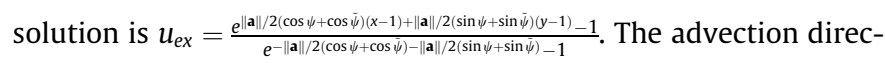
tion implied by the advection vector a is $\psi$, and $\tilde{\psi}$ denotes some flow direction. Such a function is an exact solution of the governing equation (first equation of (1)). In comparison with the example 

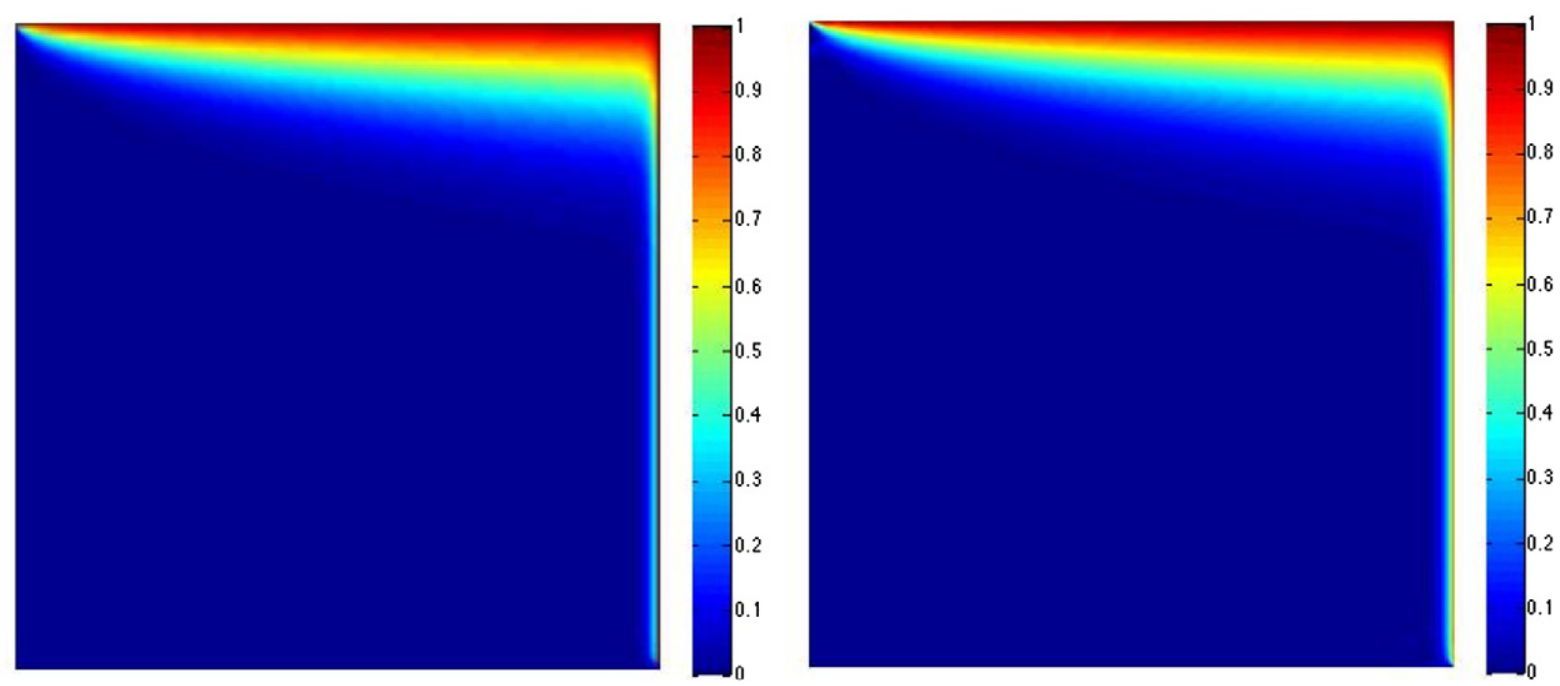

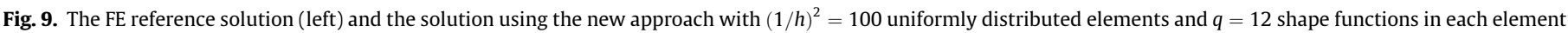
(right) for the problem of Section 5.3 and $\|\mathbf{a}\|=10^{2}$.
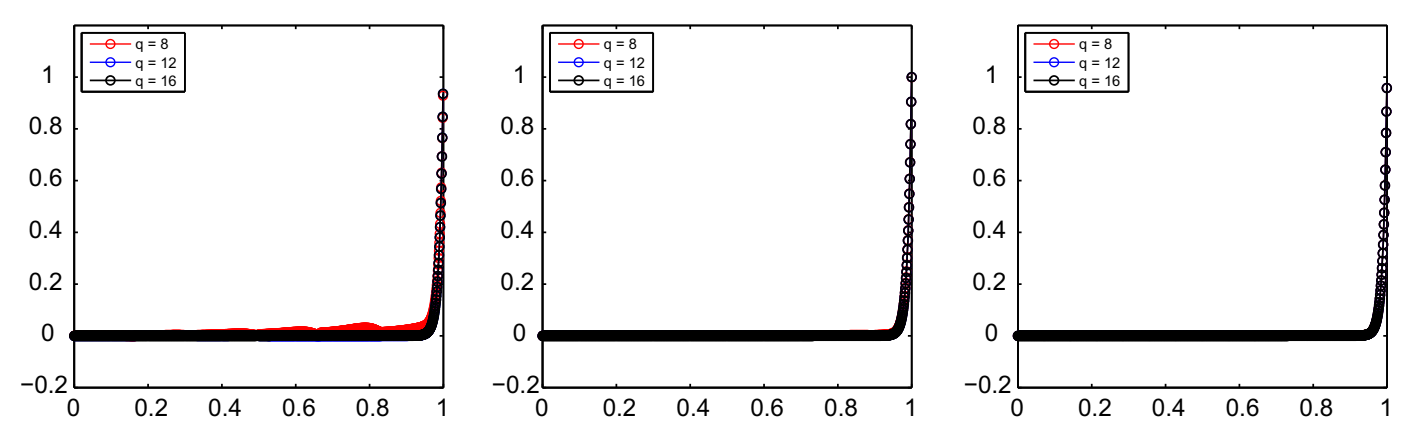

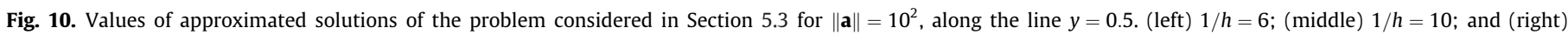
$1 / h=14$.
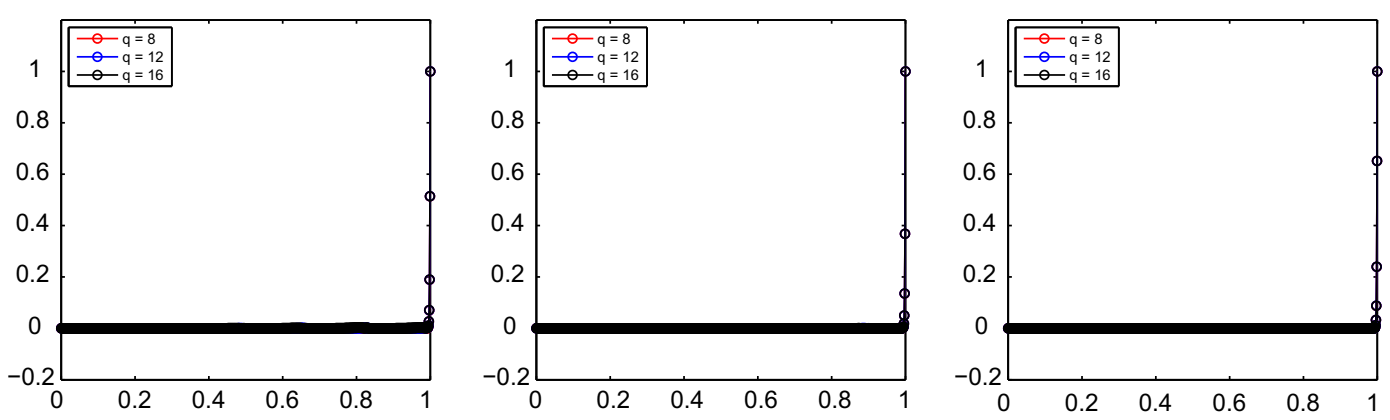

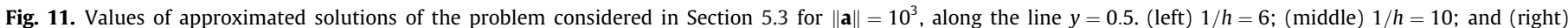
$1 / h=14$.

considered in Section 5.1, the "flow" of the solution is not oriented on the direction of $\mathbf{a}$. This example, too, was used in [16] to compare different methods, and it is interesting here because $u_{e x}$ is no longer in the span of $S_{E, a d}^{q}$ (see (11)), except for certain values of $q$. Three different values of $\|\mathbf{a}\|$ were tested: $10^{2}, 10^{3}$ and $10^{6}$. Without loss of generality, $\psi=0$ and $\tilde{\psi}=\pi / 8$ were chosen. The $h$-convergence curves were described as functions of $\frac{1}{h}$ (which corresponds to the number of elements along an edge of $\Omega$ ). The $q$-convergence curves were described as functions of $q$ (which represents the number of shape functions of an element $\Omega_{E}$ ). Each curve was plotted using the relative $L^{2}$-norm between the approximate solution and $u_{e x}$. Here $q$ was not chosen to be equal to 16 , which would have made the exact solution be in the span of $S_{E, a d}^{q}$.

Figs. 2-4 show the $h$-convergence curves for $\|\mathbf{a}\|=10^{2},\|\mathbf{a}\|=$ $10^{3}$ and $\|\mathbf{a}\|=10^{6}$ for different values of $q$. Figs. 5-7 show the $q$ convergence curves for $\|\mathbf{a}\|=10^{2},\|\mathbf{a}\|=10^{3}$ and $\|\mathbf{a}\|=10^{6}$ for different values of $\frac{1}{h}$.

Figs. 2-4 show that with increasing values of $\frac{1}{h}$, our approach converges toward the exact solution, but the convergence rate is very slow. Indeed, the slope of the curves is always very small 

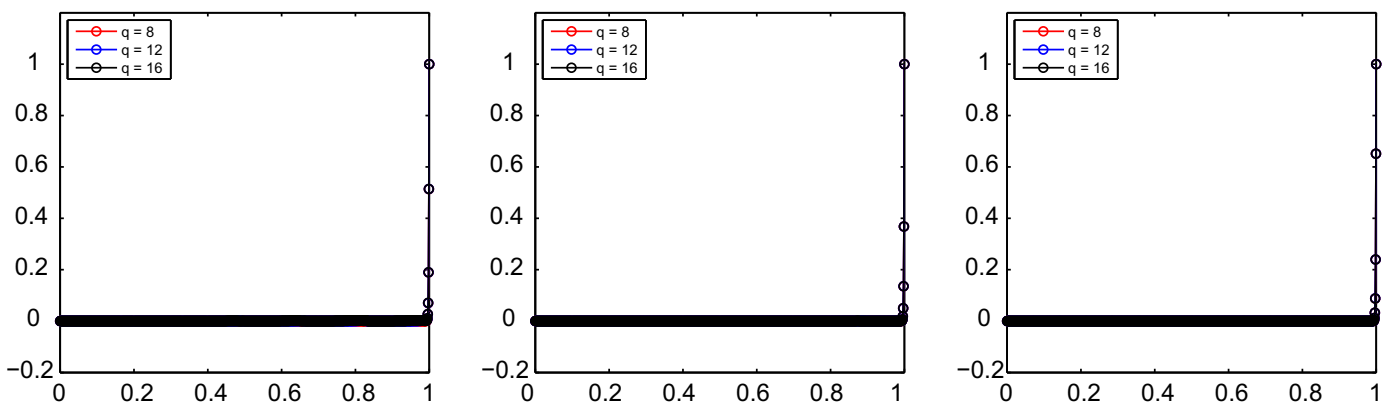

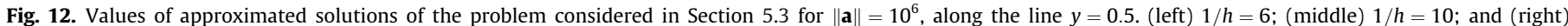
$1 / h=14$.

and, in certain cases, almost equal to zero (see Fig. 3). However, the good performance of the method becomes apparent when $\frac{1}{h}$ is fixed and $q$ increases. Indeed, by choosing $q=6, q=10$ and $q=14$ successively, the relative errors decrease each time by an order of magnitude (and sometimes two).

That remark can be made about the $q$-convergence curves of Figs. 5-7. Indeed, in these figures, an excellent convergence rate of the curves can be observed systematically, which makes the proposed method very advantageous for solving this type of problem. For $\|\mathbf{a}\|=10^{3}$ and $\|\mathbf{a}\|=10^{6}$ the curves are almost identical, which confirms the lack of interest of the $h$-approach observed in the previous section. The same behavior (the $q$-convergence is significantly better than the $h$-convergence) had already been reported with medium-frequency numerical strategies using plane waves to solve Helmholtz equations (see $[15,12,19]$ ). Of course, this conclusion is valid only for an example such as this one whose exact solution is exponential.

Finally, in Fig. 7, one can observe an interruption in the convergence at $\|\mathbf{a}\|=10^{6}$. As pointed out previously in Section 5.1, this is due to numerical difficulties related to some values of $q$ and that particular value of $\|\mathbf{a}\|$, leading to poor conditioning of the matrix associated with the problem. Nevertheless, the accuracy remains acceptable.

\subsection{The boundary layer problem with constant Dirichlet boundary conditions}

Finally, in order to show the efficiency of the proposed numerical strategy, let us consider the problem with constant Dirichlet boundary conditions described in Fig. 8. As far as the authors know, no exact solution for this problem is available. However, as it presents strong outflow boundary layers and discontinuous Dirichlet data, it represents a draconian benchmark.

In order to assess the performance of our approach and as no exact solution is available, we used the following indicator associated with the quantity:

$$
\begin{aligned}
I_{e r r}(\square)= & \int_{\partial \boldsymbol{\Omega}}\|\mathbf{a}\|^{2}|\square|^{2} d \partial \Omega \\
& +\frac{1}{2}\left(\sum_{\Gamma_{E E^{\prime}}} \int_{\Gamma_{E E^{\prime}}}\|\mathbf{a}\|^{2}\left|\square_{E}-\square_{E^{\prime}}\right|^{2}+\left|\nabla \square_{E} \cdot \mathbf{n}_{\mathbf{E}}+\nabla \square_{E^{\prime}} \cdot \mathbf{n}_{E^{\prime}}\right|^{2} d \Gamma\right)
\end{aligned}
$$

and we used the relative error indicator $\frac{\operatorname{lerr}_{\text {err }}\left(u-u_{e x}\right)}{I_{e r r}\left(u_{e x}\right)}$. One can see that this error indicator quantifies the verification of the boundary conditions and the continuity across the interfaces between elements, which is interesting in this case because the governing equation is satisfied exactly in the numerical solution and only the boundary conditions and the continuity at the interfaces are approximated. Obviously, this indicator is equal to zero for $u_{e x}$. Therefore, the proposed error indicator should decrease toward zero when the numerical approximation is refined.

Fig. 9 shows the responses for $\|\mathbf{a}\|=10^{2}$ obtained with the classical $Q_{1}$ finite elements (computed on a uniform mesh with 10,000 elements, then free from any spurious oscillation) and the proposed method with $(1 / h)^{2}=100$ uniformly distributed elements and $q=12$ shape functions in each element. One can observe that the two solutions are almost the same, either in shape or in amplitude. This simple comparison shows the capacity of the proposed method to converge toward a good solution for that problem where no exact analytical solution exists.

Furthermore, Figs. 10-12 show the cross-sections of the values of the proposed numerical solutions for $y=0.5$, for $\|\mathbf{a}\|=10^{2}, 10^{3}$ and $10^{6}, 1 / h=6,10,14$ and $q=8,12$ and 16 . This cross-section is located in the middle of the considered domain, and aligned with the direction of vector a, consequently showing the draconian boundary layer near $x=1$. Then, it is a relevant indication for assessing the stability of the proposed method. As one can see, no oscillation appear in the computed solution, even for very high Peclet numbers, which shows a relatively good stability of the method for that class of problems.

Figs. 13 and 14 show the $h$-convergence curves of the proposed approach for $\|\mathbf{a}\|=10^{2}$ and $\|\mathbf{a}\|=10^{3}$. The relative error indicator (12) was used to assess the quality of the solutions. One can see that the error indicator decreases when the number of DOFs increases, which seems logical and shows the relevance of the error indicator (12) for assessing the quality of the solution. Further-

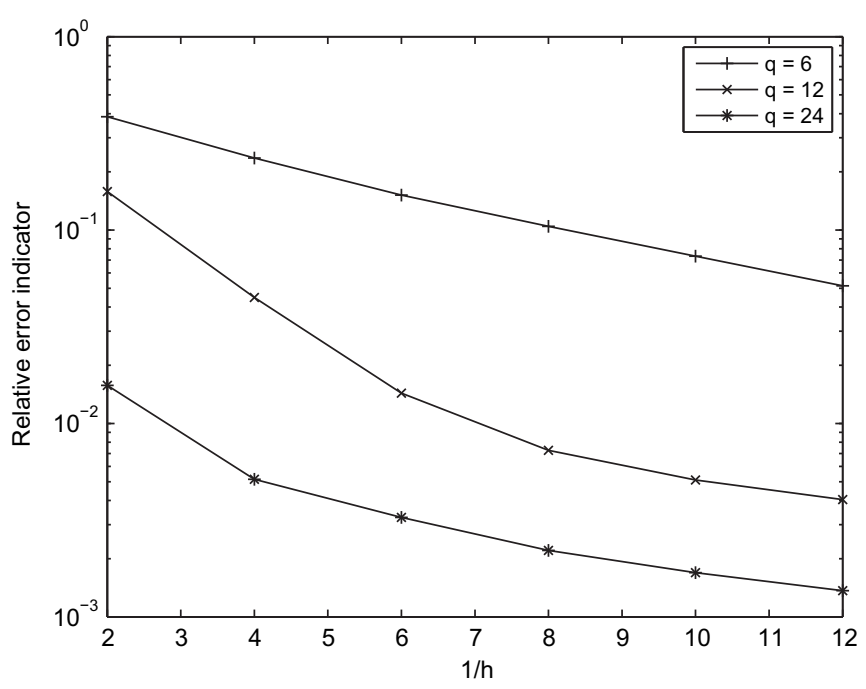

Fig. 13. $h$-convergence curves of the relative error indicator (12) for the example of Section 5.3 with $\|\mathbf{a}\|=10^{2}$. 


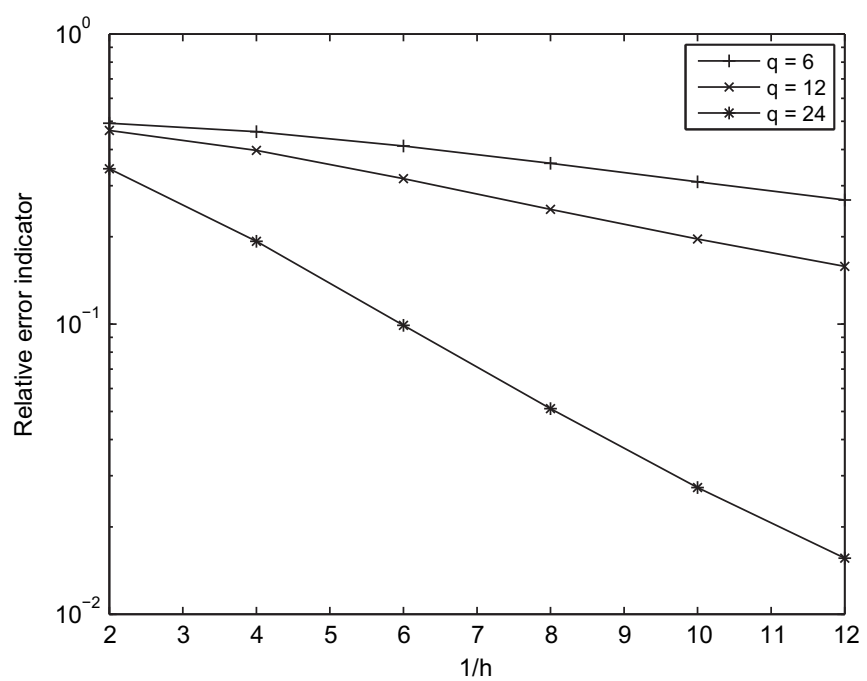

Fig. 14. $h$-convergence curves of the relative error indicator (12) for the example of Section 5.3 with $\|\mathbf{a}\|=10^{3}$

more, the greater the number of shape functions used in each element, the better the convergence. Indeed, with $q$ fixed, the three convergence curves decrease thanks to $\frac{1}{h}$ (see Figs. 13 and 14).

Figs. 15 and 16 show the $q$-convergence curves of the proposed approach for $\|\mathbf{a}\|=10^{2}$ and $\|\mathbf{a}\|=10^{3}$. As expected with the error indicator (12) and pointed-out above, the convergence curves decrease according to the number of DOFs. The fact that the convergence curves decrease rapidly shows the interest of the proposed approach for finding approximate solutions of advection-diffusion problems.

For this example, the interest of choosing an $h$-refinement vs. a $q$-refinement is illustrated in Table 2 . This table shows the value of the error indicator (12) for $\|\mathbf{a}\|=10^{2}$ and $\|\mathbf{a}\|=10^{3}$ with different choices of $\frac{1}{h}$ and $q$, keeping the total number of DOFs approximately equal to 800 each time. One can see that regardless of $\|\mathbf{a}\|$ a discretization with only a few elements, but many shape functions, is the most efficient. In fact, in all cases, $1 / h=6$ and $q=22$ lead to the smallest error indicator. Thus, as observed in Section 5.2, the $q$ refinement is preferable, at least for the example considered.

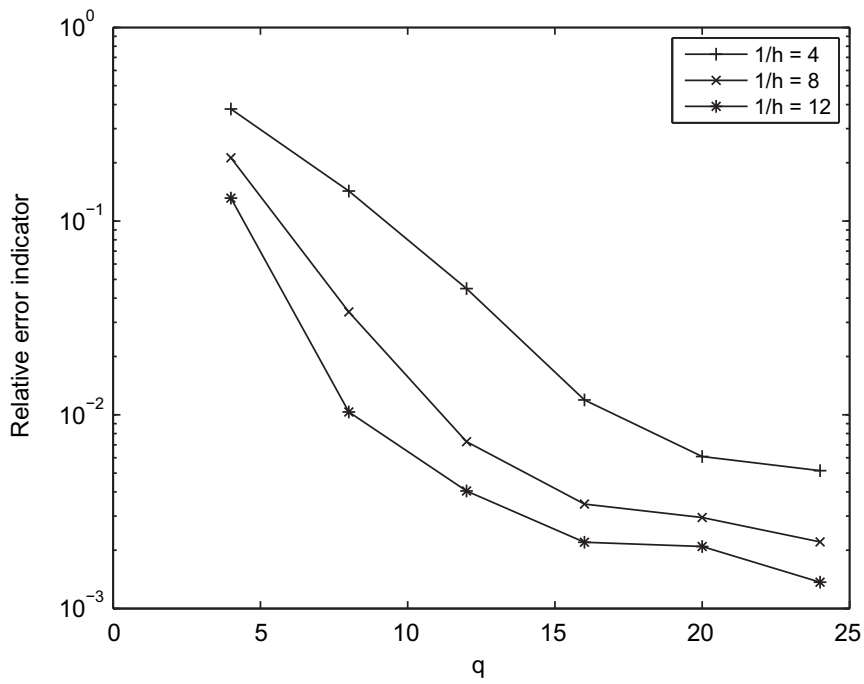

Fig. 15. $q$-convergence curves of the relative error indicator (12) for the example of Section 5.3 with $\|\mathbf{a}\|=10^{2}$.

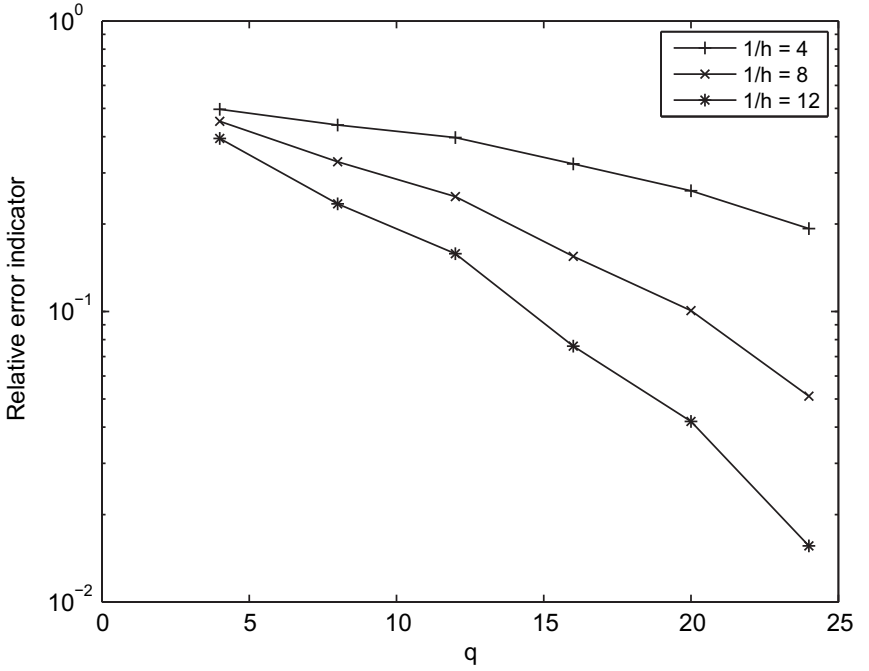

Fig. 16. $q$-convergence curves of the relative error indicator (12) for the example of Section 5.3 with $\|\mathbf{a}\|=10^{3}$.

Table 2

The relative error indicator (12) for the problem of Section 5.3 using different discretizations $(1 / h$ being the number of elements along each edge and $q$ the number of shape functions per element) leading, in each case, to about 800 DOFs.

\begin{tabular}{llll}
\hline$\|\mathbf{a}\|$ & $1 / h=6, q=22$ & $1 / h=10, q=8$ & $1 / h=14, q=4$ \\
\hline $10^{2}$ & 0.004 & 0.018 & 0.106 \\
$10^{3}$ & 0.115 & 0.277 & 0.364 \\
\hline
\end{tabular}

\section{Conclusion}

The VTCR was introduced in [1] to solve medium-frequency problems. It is based on a new variational formulation of the problem which allows the shape functions to be discontinuous across interelement boundaries. This was very interesting because plane waves could then be used as shape functions. The choice of such functions which verify the governing equation gave the strategy an excellent convergence rate.

In this paper, this idea was extended to 2-D advection-diffusion problems with constant flow velocity vectors. A dedicated variational formulation was derived and some exact solutions of the governing equation, consisting of exponential terms with sharp gradients, were used as shape functions. Three numerical examples were presented. The convergence rates were found to be excellent compared to the classical Galerkin approach. These examples showed that it is easy to implement a systematic procedure for designing elements and shape functions. They also showed that $q$-convergence works better than $h$-convergence, at least for the examples considered. All things considered, the performance of this new approach for the calculation of transport problems at high Péclet numbers appears promising.

Our future works will focus on the study of more complex problems with variable flow velocity vectors.

\section{References}

[1] P. Ladevèze, A new computational approach for structure vibrations in the medium frequency range, CR Acad. Sci. Paris 322 (IIb) (1996) 849-856.

[2] J. Donera, A. Huerta, Finite element methods for flow problems, Ed Wiley, 2003.

[3] A.N. Brooks, T.J.R. Hughes, Streamline upwind/Petrov-Galerkin formulations for convection dominated flows with particular emphasis on the 
incompressible Navier Stokes equations, Comput. Math. Appl. Mech. Engrg. 32 (1982) 199-259.

[4] A. Corsini, F. Rispoli, A. Santoriello, A quadratic Petrov-Galerkin formulation for advection- diffusion-reaction problems in turbulence modelling, J. Comput. Appl. Mech. 5 (2004) 237-249.

[5] T.J.R. Hughes, L.P. Franca, G.M. Hulbert, A new finite element formulation for computational fluid dynamics: VIII. The Galerkin/least-squares method for advective-diffusive equations, Comput. Methods Appl. Mech. Engrg. 73 (2) (1989) 173-189.

[6] I. Harari, T.J.R. Hughes, Galerkin/least squares finite element methods for the reduced wave equation with non-reflecting boundary conditions in unbounded domains, Comput. Methods Appl. Mech. Engrg. 98 (1992) 411-454.

[7] L.P. Franca, S.L. Frey, T.J.R. Hughes, Stabilized finite element methods, I. Application to the advective-diffusive model, Comput. Methods Appl. Mech. Engrg. 95 (1992) 253-276.

[8] L.P. Franca, C. Farhat, Bubble functions prompt unusual stabilized finite element methods, Comput. Methods Appl. Mech. Engrg. 123 (1995) 299308.

[9] F. Brezzi, A. Russo, Choosing bubbles for advection-diffusion problems, Math Models Methods Appl. Sci. 4 (4) (1994) 571-587.

[10] F. Brezzi, D. Marini, A. Russo, Applications of the pseudo residual-free bubbles to the stabilization of convection-diffusion problems, Comput. Methods Appl. Mech. Engrg. 166 (1998) 51-63.

[11] I. Babushka, J.M. Melenk, The partition of unity method, Int. J. Numer. Methods Engrg. 40 (1997) 727-758.
[12] C. Farhat, I. Harari, L.P. Franca, The discontinuous enrichment method, Comput. Methods Appl. Mech. Engrg. 190 (2001) 6455-6479.

[13] D. Wang, R. Tezaur, J. Toivanen, C. Farhat, Overview of the discontinuous enrichment method, the ultra-weak variational formulation, and the partition of unity method for acoustic scattering in the medium frequency regime and performance comparisons, Int. J. Numer. Methods Engrg. 89 (2012) 403-417.

[14] P. Ladevèze, L. Arnaud, P. Rouch, C. Blanzé, The variational theory of complex rays for the calculation of medium frequency vibrations, Engrg. Comput. 18 $(1-2)(2001)$ 193-214.

[15] H. Riou, P. Ladevèze, B. Sourcis, The multiscale VTCR approach applied to acoustics problems, J. Comput. Acoust. 16 (4) (2008) 487-505.

[16] I. Kalashnikova, C. Farhat, R. Tezaur, A discontinuous enrichment method for the finite element solution of high Peclet advection diffusion problems, Finite Elements Anal. Design 45 (2009) 238-250.

[17] L. Kovalevsky, P. Ladevèze, H. Riou, M. Bonnet, The variational theory of complex rays for three-dimensional Helmholtz problems, J. Comput. Acoust. accepted for publications, http://dx.doi.org/10.1142/S0218396X1250021X.

[18] I. Harari, L.P. Franca, S.P. Oliveira, Streamline design of stability parameters for advection-diffusion problems, J. Comput. Phys. 171 (2001) 115-131.

[19] T. Strouboulis, I. Babuska, R. Hidajat, The generalized finite element method for Helmholtz equation: theory, computation and open problems, Comput. Methods Appl. Mech. Engrg. 195 (2006) 4711-4731.

[20] C. Farhat, I. Kalashnikova, R. Tezaur, A higher-order discontinuous enrichment method for the solution of high Peclet advection-diffusion problems on unstructured meshes, Int. J. Numer. Methods Engrg. 81 (2010) 604-636. 